\title{
Conductivity Recovery Formula Around Point Electrodes in Electrical Impedance Tomography
}

\author{
Agah D. Garnadi
}

31 December 2017

\begin{abstract}
We consider the inverse electrical impedance problem in the case of piecewise constan conductivities with the currents injected at adjacent point electrodes and the resulting voltages measured between the remaining electrodes.

Adapting the linear functional strategy from related problem in aquifer transmittivity, the conductivity can be recovered on the zone of a domain containing the point electrodes. Such reconstruction method is needed as a preliminary step before full reconstruction of conductivity inside the domain from boundary measurements, as done in electrical impedance tomography.

Keywords: Conductivity Recovery, Electrical Impedance Tomography, Linear Functional Techniques.
\end{abstract}

\section{Electrical Impedance Tomography as parameter identifica- tion problem}

In the study of a direct electrical current in a bounded object $\Omega$, the governing equation is the following elliptic partial differential equation:

$$
-\nabla(\sigma \nabla u)=q \quad \text { in } \quad \Omega
$$

where $\Omega \in R^{d},\{d=2,3\}$ is a bounded domain, $\sigma: \Omega \rightarrow R$ denotes the conductivity coefficient, $u: \Omega \rightarrow R$ is electrical potential, $q:=I\left(\delta\left(r-s_{i}\right)-\delta\left(r-s_{j}\right)\right)$ is current injection pair through point electrode at $s_{i, j}, i \neq j, 1 \leq i, j, \leq N$, where $N$ denoting the number of electrodes. When the current source term $q$ and the coefficient $\sigma$ is given, with an appropriate prescribed boundary information for solution $u$ so that the direct (forward) problem of $u$ is uniquely solvable in $\Omega$. Conversely, the recovery information about the coefficient of conductivity $\sigma$, given the pair $(q, g)$ 
is given, where $g\left(r_{k}\right):=u\left(r_{k}\right)$ is measured potential at point electrodes at $r_{k}$ due to current injection pattern $q$, is an inverse problem with $\sigma$ being sensitive due to perturbation in $u$ and $q$. The recovery of $\sigma$ from point electrodes measurements $u$ and $q$, utilizing the equation (1), that is called Electrical Impedance Tomography with point electrode models.

\section{The Chow-Anderssen Ansatz [3]}

The essentials ill-posedness in the recovery of information about the conductivity $\sigma$ from measurements of $u$ and $q$ relates to the fact that the observed electrical potential data $u$ must be differentiated. This is clear from the structure of equation (1). The essence of the ansatz involves four steps:

a. the reformulation of the partial differential equations model in terms of a weak form,

b. the application of integration by parts to move the differentiation of the observed potential onto the analytic test function,

c. the localization of the resulting linear functional representation for the conductivity to a subregion surrounding the point electrodes where the conductivity is assumed to be constant, and

d. the choice of the shape of the localization and the form of test function that facilitates the computational performance of the resulting algorithm.

As explained in Chow and Anderssen [4] in terms of the notation of equation (1) and the application of steps (a)-(d) of the ansatz, various linear functional formulas for the estimation of $\sigma$ on the subregion $\Lambda \subset \Omega$ can be derived which depends on the assumed regularity of the solution $u$ of equation (1) and the test function $v$.

One of the schemes to estimate $\sigma$ provided in [3] is used here, i.e.

$$
\hat{\sigma}=-\frac{\int_{\Lambda} q v d x}{\int_{\Lambda} u \cdot \Delta v d x}, \quad u \in L^{2}(\Lambda), \quad v \in H_{0}^{2}(\Lambda) .
$$

The special form that it take is a direct exploitation of the assumtion that $\sigma$ is a constant in the subregion $\Lambda$. In this scheme, through an integration by parts in conjunction with the regularity imposed on the test function $v$, the evaluation of $\hat{\sigma}$ does not involve a differentiation of $u$ since it has been transferred to $v$. 


\section{The Recovery Formula}

In practice, for given current injection $q:=I\left(\delta\left(r-s_{i}\right)-\delta\left(r-s_{j}\right)\right)$ is current injection pair through point electrode at $s_{i, j}$, let $r_{m}$ is measurement point position such that $r_{m} \subset \Lambda$, supp $(q) \cap \Lambda \neq \emptyset$, and we choose $v$ is the solution of the following:

$$
\Delta v=\delta\left(r-r_{m}\right),\left.v\right|_{\partial \Lambda}=0,
$$

So, the scheme (2) resulted in:

$$
\hat{\sigma}=-\frac{\int_{\Lambda} q v d x}{\int_{\Lambda} u \cdot \Delta v d x}=-\frac{\int_{\Lambda} q v d x}{u\left(r_{m}\right)} .
$$

$$
\hat{\sigma}=- \begin{cases}\frac{I\left(v\left(s_{i}\right)-v\left(s_{j}\right)\right)}{u\left(r_{m}\right)}, & s_{i}, s_{j} \in \Lambda, \\ \frac{I v\left(s_{i}\right)}{u\left(r_{m}\right)}, & s_{i} \in \Lambda, s_{j} \notin \Lambda, \\ \frac{-I v\left(s_{j}\right)}{u\left(r_{m}\right)}, & s_{i} \notin \Lambda, s_{j} \in \Lambda .\end{cases}
$$

Hence, we able to recover the conductivity arounds measurement point at $r_{m}$.

\section{Remarks}

- Note that the recovery formula also valid for general case of current patterns $q$ under point electrode models in EIT, furthermore the assumption that the problem is under homogeneous background can be ruled out.

- In [4] was proposing adaptive methods, where adaptivity is worked out in shrinking zones manners. However, in the case of EIT with PEM, the techniques can be adapted in reverse by starting from a point expands into a larger constant zonation.

\section{References}

[1] R.S. Anderssen \& S.S. Chow, 1991, Resolving the transmissivity zonation in confined aquifer, Mini Conference in Inverse problems, Proc. CMA Australian National University, pp 247-261.

[2] R.S. Anderssen \& C. Dietrich, 1987, The inverse problem of aquifer transmissivity identification, Research Report CMA-R01-87 Centre for Mathematical Analysis Australian National University, pp 247-261.

[3] R.S. Anderssen \& B.P. Lamichhane, 2011, Piecewise Constant Aquifer Parameter Identification Recovery, in MODSIM2011, 19th International Congress of Modelling and Simulation, pp 364-370. 
[4] S.S. Chow \& R.S. Anderssen, 1991, Determination of the transmissivity zonation using a linear functional strategy, Inverse problems, $7(6)$, pp 841-851. 